\title{
SCIENTIFIC REPORTS

\section{OPEN Modified creatinine index and risk for long-term infection-related mortality in hemodialysis patients: ten-year outcomes of the Q-Cohort Study}

\begin{abstract}
Hokuto Arase ${ }^{1}$, Shunsuke Yamada ${ }^{1}$, Hiroto Hiyamuta ${ }^{1}$, Masatomo Taniguchi ${ }^{2}$, Masanori Tokumoto ${ }^{3}$, Kazuhiko Tsuruya ${ }^{4}$, Toshiaki Nakano ${ }^{1 *}$ \& Takanari Kitazono $^{1}$

Modified creatinine ( $\mathrm{Cr}$ ) index, calculated by age, sex, pre-dialysis serum $\mathrm{Cr}$ concentration, and $\mathrm{Kt} / \mathrm{V}$ for urea, is an indicator of skeletal muscle mass in hemodialysis (HD) patients. It remains unknown whether the modified $\mathrm{Cr}$ index predicts infection-related mortality in this population. We investigated the association between the modified $\mathrm{Cr}$ index and infection-related mortality. A total of 3046 patients registered in the Q-Cohort Study, a multicenter, observational study of HD patients, were analyzed. Associations between sex-specific quartiles (01-04) of the modified $\mathrm{Cr}$ index and the risk for infectionrelated mortality were analyzed by Cox proportional hazard model. During a median follow-up of 8.8 years, 387 patients died of infection. The estimated risk for infection-related mortality was significantly higher in the lower quartiles (Q1, Q2, and Q3) than in the highest quartile (Q4) as the reference group (hazard ratios and $95 \%$ confidence intervals [CI]: 01, 2.89 [1.70-5.06], 02, 2.76 [1.72-4.62], and O3, 1.79 [1.12-2.99]). The hazard ratio $(95 \% \mathrm{Cl})$ for a $1 \mathrm{mg} / \mathrm{kg} /$ day decrease in the modified $\mathrm{Cr}$ index was $1.18(1.09-1.27, P<0.01)$ for infection-related mortality. In conclusion, a lower modified $\mathrm{Cr}$ index is associated with an increased risk for long-term infection-related mortality in the HD population.
\end{abstract}

Hemodialysis (HD) patients have a higher mortality risk than the general population, and infectious disease is a major cause of mortality in patients on maintenance $\mathrm{HD}^{1}$. Among the risk factors for infectious disease in this population, malnutrition or protein energy wasting (PEW) is regarded as a critical pathophysiology ${ }^{2,3}$. Malnutrition frequently compromises HD patients and involves decreased skeletal muscle mass and function, termed sarcopenia ${ }^{2}$, eventually leading to increased morbidity and mortality ${ }^{4-6}$. These complex pathologies, involving malnutrition, inflammation, and increased risk for morbidity and mortality, have been integrated into the concept of "malnutrition-inflammation complex syndrome" (MICS)

In 1995, Canaud et al. first proposed the creatinine (Cr) index, which is a convenient indicator of the skeletal muscle mass of $\mathrm{HD}$ patients ${ }^{7}$. The original $\mathrm{Cr}$ index was derived from $\mathrm{Cr}$ kinetic modeling, and these authors have recently proposed a more simplified version of the $\mathrm{Cr}$ index requiring only age, sex, pre-dialysis serum $\mathrm{Cr}$ concentration, and Kt/V for urea, which is now termed the "modified Cr index" ${ }^{8}$. Medical practitioners can readily and cost-effectively evaluate the skeletal muscle mass of HD patients using this modified Cr index. Furthermore, we recently reported that a lower modified $\mathrm{Cr}$ index was significantly associated with higher risks for bone fracture, heart disease, and all-cause mortality from the 4-year follow-up data of the Q-Cohort Study ${ }^{9,10}$. A decrease in skeletal muscle mass reflects the MICS which is considered to be a critical underlying cause of these diverse outcomes. Therefore, we hypothesized that decreased skeletal muscle mass can also predict a risk for infectious disease in HD patients. However, the association between the modified $\mathrm{Cr}$ index and infection-related mortality in the HD population remains unclear.

${ }^{1}$ Department of Medicine and Clinical Science, Graduate School of Medical Sciences, Kyushu University, Fukuoka, Japan. ${ }^{2}$ Fukuoka Renal Clinic, Fukuoka, Japan. ${ }^{3}$ Department of Internal Medicine, Fukuoka Dental College, Fukuoka, Japan. ${ }^{4}$ Department of Nephrology, Nara Medical University, Nara, Japan. *email: toshink@med.kyushu-u.ac.jp 
The aim of the present study was to investigate the relationship between estimated skeletal muscle mass, shown by the modified $\mathrm{Cr}$ index, and long-term infection-related mortality in HD patients by analyzing the 10-year follow-up data of the Q-Cohort Study. In addition, we determined the predictive value of the modified $\mathrm{Cr}$ index using c-statistics, net reclassification improvement (NRI), and integrated discrimination improvement (IDI).

\begin{abstract}
Methods
Design of the Q-Cohort study. The Q-Cohort Study was originally designed as a multicenter, longitudinal, observational study for patients with end-stage renal disease undergoing maintenance HD in Japan. The details of this study have been previously described ${ }^{10-14}$. Briefly, 3566 outpatients who were 18 years old or older and underwent regular HD therapy between December 2006 and December 2016 at 39 dialysis facilities were enrolled. All of the patients were scheduled to be followed-up until December 2016. Of the 3566 patients, 520 who had missing data for baseline parameters or outcome were excluded. Therefore, remaining 3046 patients were analyzed in the present study. The present study was performed according to the Ethics of Clinical Research (1975 Declaration of Helsinki). The study protocol was approved by the Kyushu University Hospital Institutional Review Board for Clinical Research (No. 20-31). Written informed consent was provided by all the patients prior to participation in the study. The ethics committee of all participating institutions granted approval to waive the requirement for written informed consent for the additional follow-up surveys from 2011-2016 because of the retrospective nature of this study, which is registered in the University Hospital Medical Information Network (UMIN) clinical trial registry (UMIN ID: 000000556).
\end{abstract}

Demographics and biochemical measurements. Demographic and clinical data were ascertained at the baseline. The following data were included; age, sex, presence of diabetic nephropathy, history of cardiovascular events and bone fractures, dialysis history, dialysis time, body mass index (BMI), normalized protein catabolic rate (nPCR), systolic blood pressure, cardiothoracic ratio, and the prescription of erythropoiesis-stimulating agents (ESAs), anti-hypertensives, phosphate-binders, and vitamin D receptor activators (VDRAs). Collection and measurement of samples were performed as previously described ${ }^{10}$. Blood samples were collected by vascular access just prior to initiating dialysis sessions after a 2-day inter-dialytic interval at the baseline and were analyzed to determine hematological and biochemical parameters including hemoglobin and serum concentrations of albumin, total cholesterol, C-reactive protein (CRP), urea nitrogen, $\mathrm{Cr}$, calcium, phosphate, and alkaline phosphatase. These routine parameters were measured using an auto-analyzer with standard procedures at different laboratories, depending on the location of the dialysis centers. Whole or intact PTH assays were used to determine serum parathyroid hormone (PTH) concentrations, and conversion between the two assays was conducted using the Eq. $(1)^{15}$. Payne's formula (Eq. (2)) was used to calculate corrected serum calcium concentrations ${ }^{16}$. Single-pool Kt/V for urea was used as the index of adequacy of dialysis and was calculated without residual renal function (RRF) in the present study.

Definition of outcome and covariates. Infection-related mortality was the main outcome of the present study. Definition of infection-related death in the present study was death caused by infectious diseases including respiratory infection (e.g. pneumonia), urinary tract infection (e.g. pyelonephritis), intestinal infection (e.g. enteritis), cardiac infection (e.g. endocarditis), neurologic infection (e.g. meningitis), septicemia, vascular access-related infection, and other infections ${ }^{17}$. The covariate of interest was the modified $\mathrm{Cr}$ index ${ }^{8-10}$. Here, the modified $\mathrm{Cr}$ index was calculated using the formula (3) based on age, sex, pre-dialysis serum Cr concentrations, and $\mathrm{Kt} / \mathrm{V}$ for urea ${ }^{8-10}$. We used pre-dialysis serum $\mathrm{Cr}$ concentrations that were determined on the first dialysis day of the week. In our previous independent cohort study, a close correlation between the modified $\mathrm{Cr}$ index and skeletal muscle mass determined by bioelectrical impedance analysis was validated ${ }^{9}$.

Statistical analysis. Details of the statistical method used in the present study was also described in our previous study ${ }^{10}$. All continuous parameters are given as the median (interquartile range), while categorical parameters are given as a number (percentage). Because the modified $\mathrm{Cr}$ index distributed in different ranges between sexes, the patients were classified into quartiles (Q1-Q4) based on a sex-specific modified Cr index. Trends between quartiles of modified $\mathrm{Cr}$ index were examined by the Cochran-Armitage test for categorical parameters and by the Jonckheere-Terpstra test for continuous parameters. Kaplan-Meier survival curves for infection-related mortality among sex-specific modified $\mathrm{Cr}$ index quartiles were examined by the log-rank test. Age- and sex-adjusted and multivariable-adjusted hazard ratios (HRs) and 95\% confidence intervals (CIs) for infection-related mortality were examined by the Cox proportional hazards model. Proportional hazards were graphically checked by log cumulative hazard plots for infection-related mortality among quartiles of the modified $\mathrm{Cr}$ index. Multivariable-adjusted analyses were performed using the following potential confounding factors selected based on a priori clinical judgment: age, sex, presence of diabetic nephropathy, dialysis history, nPCR, Kt/V for urea, BMI, and serum concentrations of albumin, urea nitrogen, and CRP. The restricted cubic spline model was used to plot the multivariable-adjusted association between the modified Cr index and HRs with 95\% CIs for infection-related mortality. We performed subgroup analysis to estimate the association between a decrease in skeletal muscle mass and infection-related mortality according to subgroups of risk factors. Furthermore, to evaluate the predictive value of the modified $\mathrm{Cr}$ index for infection-related mortality, we compared a basic model and a basic model with the modified Cr index by c-statistics calculated using a receiver-operating characteristic curve, NRI, and IDI. A basic model included age, sex, the presence of diabetic nephropathy, dialysis history, $\mathrm{nPCR}, \mathrm{Kt} / \mathrm{V}$ for urea, BMI, and serum concentrations of albumin, urea nitrogen, and CRP as potential risk factors. A difference was considered significant when the $P$ value $<$ 0.05. JMP version 13.2 (SAS Institute, Cary, USA) and R software version 3.0.2 (http://cran.rproject.org) were used in statistical analyses. 


\section{Results}

Baseline characteristics. Baseline characteristics among sex-specific quartiles of the modified $\mathrm{Cr}$ index were shown in Table 1. Patients with a lower modified $\mathrm{Cr}$ index were significantly older, more frequently had diabetic nephropathy and a history of cardiovascular events and bone fractures, and had a shorter dialysis history and dialysis time, lower BMI and nPCR, and higher cardiothoracic ratio than patients with a higher modified Cr index. Patients with a lower modified $\mathrm{Cr}$ index showed significantly higher serum CRP and alkaline phosphatase concentrations than patients with a higher modified $\mathrm{Cr}$ index. By contrast, patients with a lower modified $\mathrm{Cr}$ index showed significantly lower hemoglobin and serum albumin, urea nitrogen, $\mathrm{Cr}$, corrected calcium, phosphate, and PTH concentrations than patients with a higher modified $\mathrm{Cr}$ index. Prescription rates of phosphate binders and VDRAs in patients with a lower modified Cr index were significantly lower than in patients with a higher modified $\mathrm{Cr}$ index, whereas the prescription rate of ESAs was significantly higher in patients with a lower modified $\mathrm{Cr}$ index than in patients with a higher modified $\mathrm{Cr}$ index.

Association between the modified creatinine index and the nutritional marker. Because lower serum albumin and total cholesterol concentrations, lower BMI, and decreased skeletal muscle mass are included in the criteria of $\mathrm{PEW}^{18,19}$, the association between modified $\mathrm{Cr}$ index and nutritional markers was examined. The mean modified $\mathrm{Cr}$ index was significantly lower in both the lower serum albumin concentration and lower BMI groups compared with the respective higher ones (both $P<0.01$; t test, Fig. 1), while the mean modified $\mathrm{Cr}$ index was not different between the higher and lower serum total cholesterol concentration groups.

Association between the modified creatinine index and the risk for infection-related mortality. During a median observational period of 8.8 years (interquartile range: $4.1-10.0$ years), 387 patients died of infection and 1503 patients of any cause. The incidence of infection-related mortality was highest in the lowest modified Cr index quartile (Q1) (Table 2). Kaplan-Meier curves showed significantly higher incidence rates of infection-related mortality at lower modified $\mathrm{Cr}$ index quartiles than at higher modified $\mathrm{Cr}$ index quartiles $(P<0.01$; log-rank test, Fig. 2$)$. In the age- and sex-adjusted and multivariable-adjusted Cox proportional hazards models, patients with lower modified Cr index quartiles (Q1, Q2, and Q3) displayed higher adjusted HRs for the incidence rates of infection-related mortality compared with the reference group (Q4) (Table 3). The HR $(95 \%$ CI) for a $1 \mathrm{mg} / \mathrm{kg} /$ day decrease in the modified Cr index was $1.18(1.09-1.27, P<0.01)$ for infection-related mortality. The continuous multivariable-adjusted association between the modified $\mathrm{Cr}$ index and infection-related mortality was also shown by a restricted cubic spline curve. The HRs for infection-related mortality increased incrementally with a decrease in the modified Cr index (Fig. 3).

Subgroup analysis. To evaluate the heterogeneity of the association between the modified $\mathrm{Cr}$ index and baseline parameters regarding infection-related mortality, subgroup analyses were performed. The association between a lower modified $\mathrm{Cr}$ index and infection-related mortality was reduced in patients with a shorter dialysis history compared with patients with a longer dialysis history ( $P$ for interaction $<0.001$ ) (Fig. S1).

Predictive value of the modified creatinine index. To evaluate the predictive value of the modified $\mathrm{Cr}$ index, we compared c-statistics, NRI, and IDI for infection-related mortality between the basic model with and without the modified Cr index. Although c-statistics and IDI for infection-related mortality did not significantly increase by the addition of the modified $\mathrm{Cr}$ index to the basic model, the NRI for infection-related mortality showed a slightly but significantly better performance in the basic model with the modified $\mathrm{Cr}$ index than without it (Fig. S2 and Table S1).

\section{Discussion}

In the present cohort study of patients on maintenance HD, we demonstrated that the modified Cr index was significantly associated with nutritional markers including serum albumin concentration and BMI, both of which are included in the PEW criteria. Furthermore, we demonstrated that a lower modified $\mathrm{Cr}$ index was significantly associated with a higher 10-year infection-related mortality, even after rigorous adjustment for potential confounding factors. Our study suggests that the modified Cr index, an indicator of skeletal muscle mass, can be used as a nutritional marker of HD patients, which predicts long-term prognosis of infectious mortality in this population.

Skeletal muscle is an essential organ that has multiple functions, not limited to producing mechanical movement or in skeletal support of the body. Demonstrating the importance of such multiple functions of skeletal muscle, accumulating evidence has revealed that sarcopenia is a strong risk factor for adverse events in HD patients ${ }^{5,20}$. One of the reasonable explanations for the association between a lower modified $\mathrm{Cr}$ index and infection-related mortality is the multifaceted function of skeletal muscle on immunity. First, skeletal muscle is the most important organ that induces thermogenesis ${ }^{21}$. When patients contract an infectious disease, they strengthen immunocompetence by raising body temperature mainly with frequent muscle contraction during shivering ${ }^{22}$. Hyperthermia inhibits viral and bacterial proliferation, while promoting leukocyte and macrophage migration and function. Skeletal muscle also plays an important role in energy storage ${ }^{23}$. In an infectious state, various types of hormones and cytokines are induced by stress and lead to the hypercatabolism of skeletal muscle and adipose tissue to manage a latent starved condition. Conversely, decreased skeletal muscle mass results in insufficient thermogenesis and energy expenditure, which is a potential cause of increased severity of infectious disease.

Another possible explanation for our results is the role of exercise-induced, skeletal muscle-derived cytokines termed myokines ${ }^{24}$. Recently, evidence from both clinical and basic research have shown protective effects of myokines in cardiovascular disease and mortality ${ }^{25,26}$. Furthermore, several studies demonstrated possible direct 


\begin{tabular}{|c|c|c|c|c|c|}
\hline & \multicolumn{5}{|c|}{ Sex-specific quartiles of modified Cr Index, mg/kg/day } \\
\hline & $\begin{array}{l}\text { Q1 }(n=759) \text { M: } \\
-20.31 \text { F: }-17.95\end{array}$ & $\begin{array}{l}\text { Q2 }(n=763) \mathrm{M}: \\
20.32-22.13 \mathrm{~F}: \\
17.96-19.43\end{array}$ & $\begin{array}{l}\text { Q3 }(n=762) \text { M: } \\
22.14-24.05 \text { F: } \\
19.44-20.97\end{array}$ & $\begin{array}{l}\text { Q4 }(n=762) \mathrm{M}: \\
\text { 24.06- F: } 20.98-\end{array}$ & $\begin{array}{l}P \text { for } \\
\text { trend }\end{array}$ \\
\hline \multicolumn{6}{|c|}{ Demographics and dialysis-related information } \\
\hline Modified Cr index (male), mg/kg/day & $19.2(18.5-19.8)$ & $21.3(20.8-21.7)$ & $23.0(22.6-23.4)$ & $25.3(24.6-26.3)$ & $<0.001$ \\
\hline Modified Cr index (Female), mg/kg/day & $16.9(16.1-17.4)$ & $18.8(18.4-19.1)$ & $20.3(19.8-20.7)$ & $22.1(21.4-23.0)$ & $<0.001$ \\
\hline Age, years & $74.9(68.8-80.9)$ & $68.3(61.9-74.5)$ & $62.2(56.6-67.1)$ & $52.6(44.3-58.7)$ & $<0.001$ \\
\hline Female sex & $309(40.7)$ & $309(40.5)$ & $310(40.7)$ & $309(40.6)$ & 0.970 \\
\hline Diabetic nephropathy & $311(41.0)$ & $268(35.1)$ & $195(25.6)$ & $103(13.5)$ & $<0.001$ \\
\hline History of cardiovascular events & $248(32.7)$ & $204(26.7)$ & $151(19.8)$ & $96(12.6)$ & $<0.001$ \\
\hline History of bone fracture & $134(17.7)$ & $73(9.6)$ & $56(7.4)$ & $41(5.4)$ & $<0.001$ \\
\hline Dialysis history, years & $2.8(0.8-6.5)$ & $4.5(1.9-10.3)$ & $7.1(3.0-12.7)$ & $8.2(4.0-14.4)$ & $<0.001$ \\
\hline Dialysis time ( $\geq 5$ hours) & $391(51.5)$ & $473(62.0)$ & $516(67.7)$ & $508(66.7)$ & $<0.001$ \\
\hline $\mathrm{Kt} / \mathrm{V}$ for urea & $1.6(1.4-1.7)$ & $1.6(1.4-1.8)$ & $1.6(1.4-1.8)$ & $1.6(1.4-1.7)$ & 0.545 \\
\hline Body mass index, $\mathrm{kg} / \mathrm{m}^{2}$ & $19.8(18.0-22.0)$ & $20.9(18.9-23.0)$ & $21.0(19.0-23.0)$ & $21.6(19.6-23.8)$ & $<0.001$ \\
\hline nPCR, g/kg/day & $0.9(0.8-1.0)$ & $0.9(0.8-1.0)$ & $1.0(0.9-1.1)$ & $1.0(0.9-1.1)$ & $<0.001$ \\
\hline Systolic blood pressure, $\mathrm{mmHg}$ & $153(138-169)$ & $154(141-170)$ & $153(140-168)$ & $151(136-165)$ & 0.020 \\
\hline Cardiothoracic ratio, $\%$ & $52.2(48.1-56.3)$ & $50.6(47.2-54.1)$ & $50.0(46.9-53.2)$ & $48.6(45.9-51.8)$ & $<0.001$ \\
\hline \multicolumn{6}{|l|}{ Blood tests } \\
\hline Hemoglobin, g/dL & $10.3(9.6-11.1)$ & $10.6(9.7-11.2)$ & $10.6(9.9-11.3)$ & $10.7(10.0-11.4)$ & $<0.001$ \\
\hline Serum albumin, $g / d L$ & $3.6(3.3-3.9)$ & $3.8(3.5-4.0)$ & $3.9(3.7-4.1)$ & $4.0(3.8-4.2)$ & $<0.001$ \\
\hline Serum total cholesterol, mg/dL & $150(128-175)$ & $152(132-179)$ & $153(131-180)$ & $151(130-176)$ & 0.507 \\
\hline Serum urea nitrogen, $\mathrm{mg} / \mathrm{dL}$ & $57(46-68)$ & $64(56-74)$ & $69(60-78)$ & $73(65-82)$ & $<0.001$ \\
\hline Serum $\mathrm{Cr}, \mathrm{mg} / \mathrm{dL}$ & $7.3(6.4-8.2)$ & $9.5(8.7-10.4)$ & $11.2(10.1-12.1)$ & $13.3(11.9-14.5)$ & $<0.001$ \\
\hline Serum C-reactive protein, $\mathrm{mg} / \mathrm{dL}$ & $0.2(0.1-0.6)$ & $0.1(0.1-0.4)$ & $0.1(0.1-0.3)$ & $0.1(0-0.2)$ & $<0.001$ \\
\hline Corrected serum Ca, mg/dL & $9.2(8.8-9.7)$ & $9.3(8.9-9.8)$ & $9.5(8.9-10.0)$ & $9.5(9.0-10.0)$ & $<0.001$ \\
\hline Serum phosphate, $\mathrm{mg} / \mathrm{dL}$ & $4.4(3.7-5.1)$ & $4.7(4.1-5.5)$ & $5.1(4.4-5.8)$ & $5.3(4.6-6.1)$ & $<0.001$ \\
\hline Serum alkaline phosphatase, U/L & $258(205-346)$ & $242(190-313)$ & $226(177-306)$ & $209(162-279)$ & $<0.001$ \\
\hline Serum PTH (intact assay), pg/mL & $85(40-155)$ & $103(44-207)$ & $106(46-215)$ & $129(57-254)$ & $<0.001$ \\
\hline \multicolumn{6}{|l|}{ Medications } \\
\hline Prescription of phosphate binders & $476(62.7)$ & $624(81.8)$ & $677(88.9)$ & 707 (92.8) & $<0.001$ \\
\hline Prescription of VDRAs & $471(62.1)$ & $544(71.3)$ & $571(74.9)$ & $569(74.7)$ & $<0.001$ \\
\hline Prescription of anti-hypertensives & $487(64.2)$ & $502(65.8)$ & $493(64.7)$ & $465(61.0)$ & 0.176 \\
\hline Prescription of ESAs & $684(90.1)$ & $649(85.1)$ & $633(83.1)$ & $594(78.0)$ & $<0.001$ \\
\hline
\end{tabular}

Table 1. Clinical background at baseline of each group stratified according to sex-specific modified creatinine index quartiles $(n=3046)$. Cr, creatinine; Ca, calcium; ESAs, erythropoiesis-stimulating agents; $\mathrm{F}$, female; $\mathrm{M}$, male; nPCR, normalized protein catabolic rate; PTH, parathyroid hormone; Q, quartile of the modified $\mathrm{Cr}$ index; VDRAs, vitamin D receptor activators. Baseline data are expressed as the median (interquartile range) or number (percentage). The Cochran-Armitage test was used to determine $\mathrm{P}$ for a trend of categorical variables. The Jonckheere-Terpstra test was used to determine $\mathrm{P}$ for a trend of continuous variables. A two-tailed P value $<0.05$ was considered statistically significant.

effects of myokines on immunity ${ }^{27}$. Although a detailed mechanism has not been clarified, the protective effects of myokines on various organs, including the immune system, should be noted.

Furthermore, we need to consider the underlying mechanism regarding the association between the modified $\mathrm{Cr}$ index and our clinical outcome other than through direct effects of skeletal muscle. In our present and previous studies, we demonstrated that the modified $\mathrm{Cr}$ index strongly correlates with nutritional and inflammatory markers, including the BMI, $\mathrm{nPCR}$, and serum albumin and CRP concentrations ${ }^{10}$. That is, these associations show that a decrease in skeletal muscle mass evidenced by a lower modified Cr index partly reflects the MICS. Many previous reports demonstrated that MICS is a strong predictor for a worse prognosis of all-cause mortality, cardiovascular events, and infectious disease in HD patients ${ }^{6,28,29}$.Therefore, our results suggest that the modified $\mathrm{Cr}$ index serves as a comprehensive indicator of MICS and of skeletal muscle mass, and has a good correlation with diverse clinically important outcomes, including infectious mortality.

Subgroup analyses demonstrated that a shorter dialysis history reduced the association between sarcopenia and infection-related mortality. One possible explanation for this heterogeneity may be that RRF was not included when calculating the modified $\mathrm{Cr}$ index. Because the serum $\mathrm{Cr}$ concentration is determined by RRF and dialysis adequacy in HD patients ${ }^{30}$, the skeletal muscle mass of patients with a shorter dialysis history, who are likely to have a higher RRF, may not be evaluated accurately by the modified Cr index. Furthermore, because medium-sized proteins, including cytokines, are difficult to eliminate in $\mathrm{HD}$, renal excretion of cytokines is also important in HD patients ${ }^{31,32}$. Therefore, the ability to eliminate inflammatory cytokines from the body may 
(A)

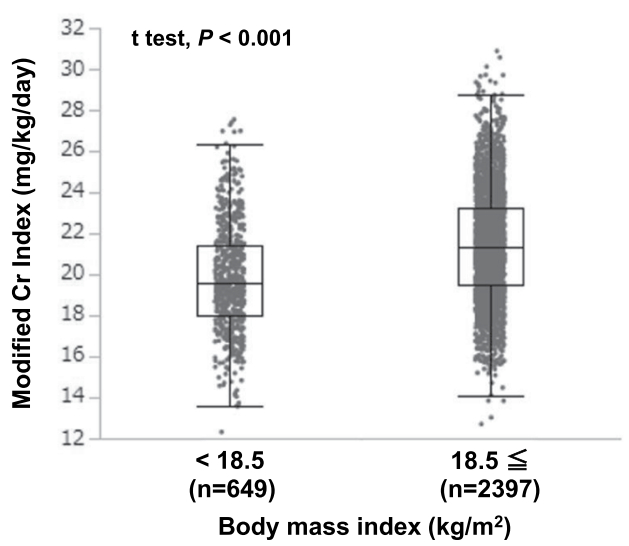

(C)

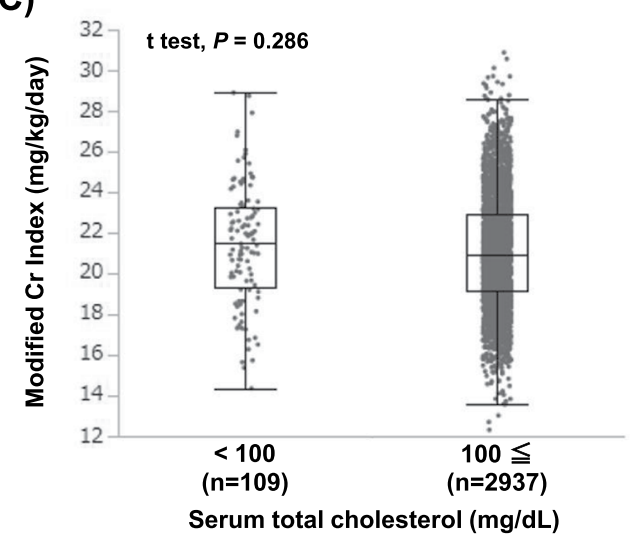

(B)

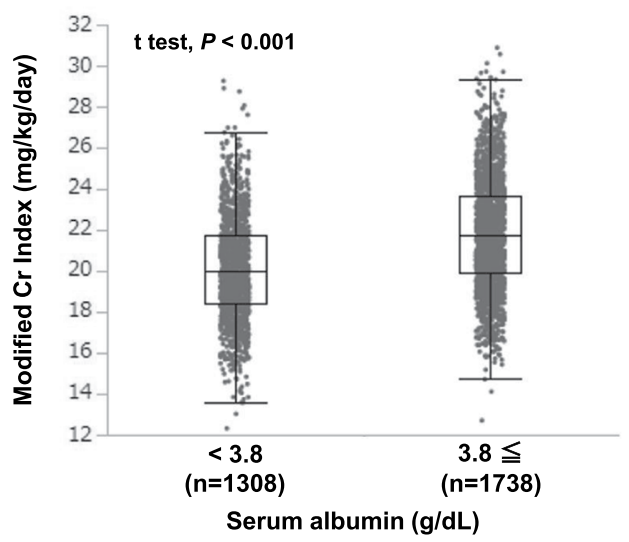

Figure 1. Distribution of the modified Cr index in each group stratified by nutritional markers including (A) body mass index, (B) serum albumin concentration, and (C) serum total cholesterol concentration. Unpaired t-test was used to analyze statistical differences. A two-tailed $P$ value $<0.05$ was considered statistically significant. Abbreviations: Cr, creatinine.

\begin{tabular}{|c|c|c|c|c|c|}
\hline & \multicolumn{5}{|c|}{ Sex-specific quartiles of modified $\mathrm{Cr}$ index, $\mathrm{mg} / \mathrm{kg} / \mathrm{day}$} \\
\hline & $\begin{array}{l}\text { Q1 }(n=759) \text { M: } \\
-20.31 \text { F: }-17.95\end{array}$ & $\begin{array}{l}\text { Q2 }(n=763) \mathrm{M}: \\
20.32-22.13 \mathrm{~F}: \\
17.96-19.43\end{array}$ & $\begin{array}{l}\text { Q3 }(n=762) \mathrm{M}: \\
22.14-24.05 \mathrm{~F}: \\
19.44-20.97\end{array}$ & $\begin{array}{l}\text { Q4 }(n=762) \mathrm{M}: \\
24.06-\mathrm{F}: 20.98-\end{array}$ & $\begin{array}{l}P \text { for } \\
\text { trend }\end{array}$ \\
\hline Infection-related death & $149(19.6)$ & $142(18.6)$ & $73(9.6)$ & $23(3.0)$ & $<0.001$ \\
\hline All-cause death & $594(78.3)$ & $471(61.7)$ & $305(40.0)$ & $133(17.5)$ & $<0.001$ \\
\hline
\end{tabular}

Table 2. Outcomes during the observation period in each group stratified according to sex-specific modified creatinine index quartiles $(n=3046)$. Cr, creatinine; F, female; $\mathrm{M}$, male; $\mathrm{Q}$, quartile of the modified $\mathrm{Cr}$ index. Outcomes during the observation period are expressed as number (percentage). The Cochran-Armitage test was used to determine $\mathrm{P}$ for the trend of outcomes. A two-tailed $\mathrm{P}$ value $<0.05$ was considered statistically significant.

be greater in patients with a higher RRF than those with a lower RRF, leading to a lesser impact of MICS on infection-related mortality in patients with a higher RRF.

Because our study has a large number of subjects with wide-ranging inclusion criteria, our data should better reflect the risk for infection-related mortality in a real-world HD population. No large-scale cohort study has ever shown an association between the modified $\mathrm{Cr}$ index and infection-related mortality in HD patients. Furthermore, we previously demonstrated the relationships between the modified Cr index and various outcomes, including bone fractures, heart disease, and all-cause mortality ${ }^{9,10}$. Although data from France was initially used to create the modified $\mathrm{Cr}$ index ${ }^{8}$, our results displayed the universal usefulness of the modified $\mathrm{Cr}$ index independent of the patients' racial group.

Our study had several limitations. First, the characteristics, including the modified Cr index, were measured only once at baseline. Therefore, misclassification bias cannot be totally excluded. Furthermore, because the modified $\mathrm{Cr}$ index might have changed during the observation period, a time-dependent model is better for survival analysis and is required in any future studies. Second, we did not classify the type of infection, whether pneumonia, urinary tract infection, or vascular access-related infection. It is possible that the modified Cr index 


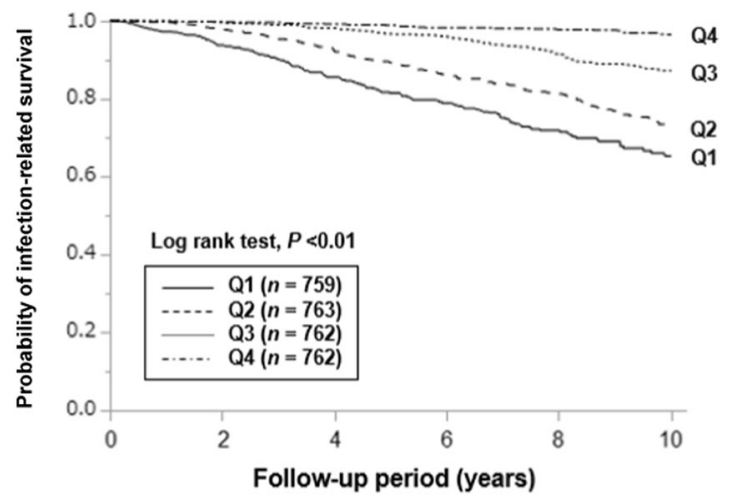

\begin{tabular}{cccccc}
\multicolumn{2}{c}{ Number at risk } & & & & \\
759 & 576 & 400 & 294 & 199 & 96 \\
763 & 684 & 574 & 457 & 358 & 226 \\
762 & 720 & 647 & 588 & 503 & 373 \\
762 & 729 & 689 & 640 & 599 & 507
\end{tabular}

Figure 2. Kaplan-Meier curves for the incidence of infection-related mortality in each group stratified by the sex-specific modified $\mathrm{Cr}$ index. Patients were stratified into sex-specific quartiles according to the modified $\mathrm{Cr}$ index. Log-rank test was used to analyze statistical differences. A two-tailed $P$ value $<0.05$ was considered statistically significant. Abbreviations: Cr, creatinine; Q, quartile of the modified Cr index.

\begin{tabular}{|l|l|l|l|l|l|l|}
\hline \multirow{2}{*}{} & \multicolumn{4}{|l|}{ Age- and sex-adjusted } & \multicolumn{4}{l|}{ Multivariable-adjusted } \\
\cline { 2 - 8 } & HR $(\mathbf{9 5} \mathbf{C I})$ & $\boldsymbol{P}$-value & $\boldsymbol{P}$ for trend & HR $\mathbf{9 5 \%} \mathbf{C I})$ & $\boldsymbol{P}$-value & $\boldsymbol{P}$ for trend \\
\hline Infection-related mortality & & & $<0.001$ & & & $<0.001$ \\
\hline Q1 & $4.31(2.66-7.26)$ & $<0.001$ & & $2.89(1.70-5.06)$ & $<0.001$ & \\
\hline Q2 & $3.59(5.59-5.93)$ & $<0.001$ & & $2.76(1.72-4.62)$ & $<0.001$ & \\
\hline Q3 & $2.09(2.25-3.48)$ & 0.002 & & $1.79(1.12-2.99)$ & 0.014 & \\
\hline Q4 & $1.00($ reference) & - & & $1.00($ reference $)$ & - & \\
\hline $\begin{array}{l}\text { Every 1-mg/kg/day decrease in the } \\
\text { modified Cr index }\end{array}$ & $1.23(1.17-1.31)$ & $<0.001$ & & $1.18(1.09-1.27)$ & $<0.001$ & \\
\hline
\end{tabular}

Table 3. Hazard ratios for Infection-related mortality in each group stratified by sex-specific modified creatinine index quartiles $(n=3046)$. CI, confidence interval; Cr, creatinine; nPCR, normalized protein catabolic rate, HR, hazard ratio; Q, quartile of the modified creatinine index. Age- and sex-adjusted and multivariable-adjusted HRs were analyzed by Cox proportional hazards risk model. The covariates for assessing infection-related mortality included age, sex, the presence of diabetic nephropathy, dialysis history, nPCR, Kt/V for urea, body mass index, and serum concentrations of albumin, urea nitrogen, and C-reactive protein. A two-tailed $P$ value $<0.05$ was considered statistically significant.

may impact differently on the different causes of infection-related mortality. Third, as previously described, RRF was not measured in the present study. RRF might affect serum $\mathrm{Cr}$ concentration and Kt/V for urea, both of which were included in the formula of the modified $\mathrm{Cr}$ index. Therefore, the modified $\mathrm{Cr}$ index might not reflect skeletal muscle mass accurately particularly in patients with preserved RRF. Fourth, a causal relationship between the modified $\mathrm{Cr}$ index and outcome could not be concluded because the study was designed as an observational study. Finally, although we adjusted for potential confounders, known and unknown residual confounders might modify our results. Despite these limitations, we believe that our results provide useful information on the association between the modified $\mathrm{Cr}$ index, skeletal muscle mass indicator, and critical clinical outcome in patients on maintenance HD.

In conclusion, our findings demonstrated that decreased skeletal muscle mass, represented by a lower modified $\mathrm{Cr}$ index, was significantly associated with a greater long-term risk for infection-related mortality in HD patients. Furthermore, the modified $\mathrm{Cr}$ index was a good predictor for these outcomes in the HD population. Future research is needed to answer the question of whether intervention to increase skeletal muscle mass is beneficial to decrease diverse life-threatening outcomes in HD patients.

\section{Equations.}

$$
\text { intact } \mathrm{PTH}(\mathrm{pg} / \mathrm{mL})=1.7 \times \text { whole PTH }(\mathrm{pg} / \mathrm{mL})
$$

corrected serum calcium $=$ serum calcium concentration $+(4-$ serum albumin concentration $)$ 


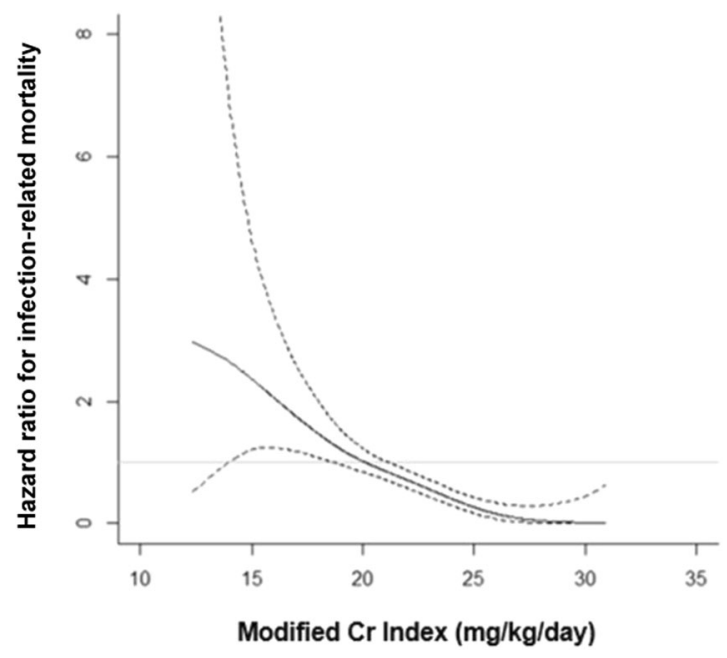

Figure 3. Multivariable-adjusted restricted cubic spline plots of the hazard ratio (HR) for infection-related mortality according to the modified $\mathrm{Cr}$ index. The solid line represents the HR and the dotted lines the 95\% confidence intervals. The multivariable-adjusted model was adjusted for age, sex, presence of diabetic nephropathy, dialysis history, normalized protein catabolic rate, $\mathrm{Kt} / \mathrm{V}$ for urea, body mass index, and serum concentrations of albumin, urea nitrogen, and C-reactive protein. Abbreviations: $\mathrm{Cr}$, creatinine.

$$
\begin{aligned}
& \text { modified } \mathrm{Cr} \text { index }(\mathrm{mg} / \mathrm{kg} / \text { day })=16.21+(1.12 \times[1 \text { if male; } 0 \text { if female }]) \\
& -(0.06 \times \text { age }(\text { years }))-(0.08 \times \text { single-pooled } \mathrm{Kt} / \mathrm{V} \text { for urea }) \\
& +(0.009 \times \text { serum } \mathrm{Cr} \text { concentration before dialysis }(\mu \mathrm{mol} / \mathrm{L}))
\end{aligned}
$$

\section{Data availability}

The datasets generated during and/or analyzed during the current study are available from the corresponding author on reasonable request.

Received: 27 August 2019; Accepted: 13 January 2020;

Published online: 27 January 2020

\section{References}

1. Nakai, S. et al. Overview of regular dialysis treatment in Japan (as of 31 December 2008). Ther Apher Dial. 14, 505-540 (2010).

2. Aparicio, M. et al. Nutritional status of haemodialysis patients: a French national cooperative study. French Study Group for Nutrition in Dialysis. Nephrol Dial Transplant. 14, 1679-1686 (1999).

3. Rocco, M. V. et al. Nutritional status in the HEMO study cohort at baseline. Am J Kidney Dis. 39, 245-256 (2002).

4. Huang, C. X. et al. Both low muscle mass and low fat are associated with higher all-cause mortality in hemodialysis patients. Kidney Int. 77, 624-629 (2010).

5. Giglio, J. et al. Association of sarcopenia with nutritional parameters, quality of life, hospitalization, and mortality rates of elderly patients on hemodialysis. J Ren Nutr. 28, 197-207 (2018).

6. Kalantar-Zadeh, K., Ikizler, T. A., Block, G., Avram, M. M. \& Kopple, J. D. Malnutrition-inflammation complex syndrome in dialysis patients: causes and consequences. Am J Kidney Dis. 42, 864-881 (2003).

7. Canaud, B. et al. Creatinine kinetic modelling: a simple and reliable tool for the assessment of protein nutritional status in haemodialysis patients. Nephrol Dial Transplant. 10, 1405-1410 (1995).

8. Canaud, B. et al. Creatinine index as a surrogate of lean body mass derived from urea $\mathrm{Kt} / \mathrm{V}$, pre-dialysis serum levels and anthropometric characteristics of haemodialysis patients. PLoS One. 9, e93286 (2014).

9. Yamada, S. et al. Modified creatinine index and the risk of bone fracture in patients undergoing hemodialysis: the Q-Cohort study. Am J Kidney Dis. 70, 270-280 (2017).

10. Arase, H. et al. Modified creatinine index and risk for cardiovascular events and all-cause mortality in patients undergoing hemodialysis: The Q-Cohort study. Atherosclerosis. 275, 115-123 (2018).

11. Taniguchi, M. et al. Does cinacalcet improve the prognosis of dialysis patients? Ther Apher Dial. 13(Suppl 1), S15-19 (2009).

12. Eriguchi, R. et al. Hyporesponsiveness to erythropoiesis-stimulating agent as a prognostic factor in Japanese hemodialysis patients: the Q-Cohort study. J Nephrol. 28, 217-225 (2015).

13. Yotsueda, R. et al. Cardiothoracic ratio and all-cause mortality and cardiovascular disease events in hemodialysis patients: the Q-Cohort study. Am J Kidney Dis. 70, 84-92 (2017).

14. Yamada, S. et al. Association between serum phosphate levels and stroke risk in patients undergoing hemodialysis: the Q-Cohort study. Stroke. 47, 2189-2196 (2016).

15. Kazama, J. J. Japanese Society of Dialysis Therapy treatment guidelines for secondary hyperparathyroidism. Ther Apher Dial. 11(Suppl 1), S44-47 (2007).

16. Payne, R. B., Little, A. J., Williams, R. B. \& Milner, J. R. Interpretation of serum calcium in patients with abnormal serum proteins. Br Med J. 4, 643-646 (1973).

17. Yamada, S. et al. Use of phosphate-binders and risk of infection-related and all-cause mortality in patients undergoing hemodialysis: the Q-Cohort Study. Sci Rep. 8, 11387 (2018). 
18. Fouque, D. et al. A proposed nomenclature and diagnostic criteria for protein-energy wasting in acute and chronic kidney disease. Kidney Int. 73, 391-398 (2008).

19. Kanazawa, Y., Nakao, T., Murai, S., Okada, T. \& Matsumoto, H. Diagnosis and prevalence of protein-energy wasting and its association with mortality in Japanese haemodialysis patients. Nephrology (Carlton). 22, 541-547 (2017).

20. Pereira, R. A. et al. Sarcopenia in chronic kidney disease on conservative therapy: prevalence and association with mortality. Nephrol Dial Transplant. 30, 1718-1725 (2015).

21. Periasamy, M., Herrera, J. L. \& Reis, F. C. G. Skeletal muscle thermogenesis and its role in whole body energy metabolism. Diabetes Metab J. 41, 327-336 (2017)

22. Theilen, H. \& Ragaller, M. Therapy of hyperthermia in sepsis and septic shock. Necessary or injurious? Der Anaesthesist. 56(949-952), 954-946 (2007).

23. Schnyder, S. \& Handschin, C. Skeletal muscle as an endocrine organ: PGC-1alpha, myokines and exercise. Bone. 80, 115-125 (2015),

24. Karstoft, K. \& Pedersen, B. K. Skeletal muscle as a gene regulatory endocrine organ. Current opinion in clinical nutrition and metabolic care. 19, 270-275 (2016).

25. He, L., He, W. Y., A, L. T., Yang, W. L. \& Zhang, A. H. Lower serum irisin levels are associated with increased vascular calcification in hemodialysis patients. Kidney Blood Press Res. 43, 287-295 (2018).

26. Han, F., Zhang, S., Hou, N., Wang, D. \& Sun, X. Irisin improves endothelial function in obese mice through the AMPK-eNOS pathway. Am J Physiol Heart Circ Physiol. 309, H1501-1508 (2015).

27. Bosma, M. et al. FNDC4 acts as an anti-inflammatory factor on macrophages and improves colitis in mice. Nat Commun. 7, 11314 (2016).

28. Kalantar-Zadeh, K. \& Kopple, J. D. Relative contributions of nutrition and inflammation to clinical outcome in dialysis patients. Am J Kidney Dis. 38, 1343-1350 (2001).

29. Zimmermann, J., Herrlinger, S., Pruy, A., Metzger, T. \& Wanner, C. Inflammation enhances cardiovascular risk and mortality in hemodialysis patients. Kidney Int. 55, 648-658 (1999).

30. Patel, S. S. et al. Serum creatinine as a marker of muscle mass in chronic kidney disease: results of a cross-sectional study and review of literature. J Cachexia Sarcopenia Muscle. 4, 19-29 (2013).

31. de Sequera, P. et al. Residual renal function in hemodialysis and inflammation. Ther Apher Dial. 21, 592-598 (2017).

32. Shafi, T. et al. Association of residual urine output with mortality, quality of life, and inflammation in incident hemodialysis patients: the Choices for Healthy Outcomes in Caring for End-Stage Renal Disease (CHOICE) Study. Am J Kidney Dis. 56, 348-358 (2010).

\section{Acknowledgements}

We would like to thank all the doctors and medical staff who participated in the Q-Cohort Study. The following personnel (institutions) participated in this trial: Takashi Ando (Hakozaki Park Internal Medicine Clinic); Takashi Ariyoshi (Ariyoshi Clinic); Kouichiro Goto (Goto Clinic); Fumitada Hattori (Nagao Hospital); Harumichi Higashi (St Mary’s Hospital); Tadashi Hirano (Hakujyuji Hospital); Kei Hori (Munakata Medical Association Hospital); Takashi Inenaga (Ekisaikai Moji Hospital); Hidetoshi Kanai (Kokura Memorial Hospital); Shigemi Kiyama (Kiyama Naika); Tetsuo Komota (Komota Clinic); Hiromasa Kuma (Kuma Clinic); Toshiro Maeda (Kozenkai-Maeda Hospital); Junichi Makino (Makino Clinic); Dai Matsuo (Hirao Clinic); Chiaki Miishima (Miishima Naika Clinic); Koji Mitsuiki (Japanese Red Cross Fukuoka Hospital); Kenichi Motomura (Motomura Naika Clinic); Sadatoshi Nakamura (Kokura Daiichi Hospital); Hidetoshi Nakamura (Kokura Daiichi Hospital); Koichi Nakashima (Ohashi Internal Circulatory Clinic); Nobumitsu Okita (Shiroishi Kyoritsu Hospital); Shinichiro Osato (Osato Jin Clinic); Sakura Sakamoto (Fujiyamato Spa Hospital); Keiko Shigematsu (Shigematsu Clinic); Kazumasa Shimamatsu (Shimamatsu Naika Iin); Yoshito Shogakiuchi (Shin-Ai Clinic); Hiroaki Takamura (Hara Hospital); Kazuhito Takeda (Iizuka Hospital); Asuka Terai (Chidoribashi Hospital); Hideyoshi Tanaka (Mojiko-Jin Clinic); Suguru Tomooka (Hakozaki Park Internal Medicine Clinic); Jiro Toyonaga (Fukuoka Renal Clinic); Hiroshi Tsuruta (Steel Memorial Yawata Hospital); Ryutaro Yamaguchi (Shiseikai Hospital); Taihei Yanagida (Saiseikai Yahata General Hospital); Tetsuro Yanase (Yanase Internal Medicine Clinic); Tetsuhiko Yoshida (Hamanomachi Hospital); Takahiro Yoshimitsu (Gofukumachi Kidney Clinic, Harasanshin Hospital); Koji Yoshitomi (Yoshitomi Medical Clinic). We thank Robert Blakytny, PhD, from Edanz Group (www. edanzediting.com/ac) for editing a draft of this manuscript. The Q-Cohort Study is supported by The Kidney Foundation, Japan (JKFB07-13, JKFB08-8, JKFB11-11) and The Japan Dialysis Outcome Research Foundation (076-02), without restriction on publications.

\section{Author contributions}

Conception and design of the study: H.A., S.Y., H.H. and M.Ta, and M.To.; generation, collection, assembly, analysis, and interpretation of data: H.A., S.Y. and H.H.; drafting and revision of the manuscript: T.N., K.T. and T.K.; approval of the final version of the manuscript: T.N.

\section{Competing interests}

The authors declare no competing interests.

\section{Additional information}

Supplementary information is available for this paper at https://doi.org/10.1038/s41598-020-58181-6.

Correspondence and requests for materials should be addressed to T.N.

Reprints and permissions information is available at www.nature.com/reprints.

Publisher's note Springer Nature remains neutral with regard to jurisdictional claims in published maps and institutional affiliations. 
(c) (i) Open Access This article is licensed under a Creative Commons Attribution 4.0 International License, which permits use, sharing, adaptation, distribution and reproduction in any medium or format, as long as you give appropriate credit to the original author(s) and the source, provide a link to the Creative Commons license, and indicate if changes were made. The images or other third party material in this article are included in the article's Creative Commons license, unless indicated otherwise in a credit line to the material. If material is not included in the article's Creative Commons license and your intended use is not permitted by statutory regulation or exceeds the permitted use, you will need to obtain permission directly from the copyright holder. To view a copy of this license, visit http://creativecommons.org/licenses/by/4.0/.

(C) The Author(s) 2020 